\title{
Discussion on the modern research and comprehensive application of bitterness
}

\author{
Zhao Hui ${ }^{1, \text { a }, \text { Miao Mingsan }}{ }^{2, \text { b, * }}$ \\ ${ }^{1}$ Pharmacological, Henan University of Chinese Medicine, Zhengzhou, China \\ ${ }^{2}$ College of Postgraduates, Henan University of Chinese Medicine, Zhengzhou, China \\ a zhaohui814@126.com, ${ }^{\mathrm{b}}$ miaomingsan@163.com \\ *corresponding author
}

Keywords: chemical composition; pharmacological action; clinical application; non-medicinal parts

Abstract: Objective: To explore the modern research and application of bitter buckwheat. Methods: Through the research on the chemical constituents, pharmacological effects, clinical application and comprehensive application of tartary buckwheat, the research direction and application value of tartary buckwheat were discussed. Results: The results showed that the active ingredient in the bitter medicinal part was high and had rich nutritional value. Conclusion: Bitter mites contain a variety of chemical components, and the rational use of bitter resources can create broad social and economic benefits.

\section{Introduction}

Tartary buckwheat is a mature seed of the genus Fagopyrum tataricum (L.) Gaertn. It is a common drug used for medicine, food and food. The bitterness was first recorded in "Shen Nongshu" and "Qi Min Yao Shu". The "Compendium of Materia Medica" records that bitterness can be "lowering the intestines, grinding the stagnation, clearing away heat and toxic wind and pain". The drug is rich in chemical components. Tartary buckwheat can be applied to the prevention and treatment of various diseases, and bitter buckwheat has been widely used as a homologous product of medicine and food.

\section{Chemical Composition}

\subsection{Flavonoids}

The buckwheat flavonoids can be extracted and purified by AB-8 macroporous adsorption resin. Under the experimental conditions, the flavonoids with higher purity can be isolated ${ }^{[1]}$. The results showed that the total flavonoids of tartary buckwheat were positive for $\alpha$-glucosidase inhibition ${ }^{[2]}$.The results of its non-medical parts showed that the tartary buckwheat bran mixed with $80 \%$ aqueous ethanol solution, extracted into the tartary buckwheat flavonoid solution, indicating that its ingredients can effectively remove the free Base, also has antioxidant capacity ${ }^{[3]}$.

\subsection{Sugar}

The extract of tartary buckwheat polysaccharide was obtained by drying at $65{ }^{\circ} \mathrm{C}$ to constant temperature and refluxing at $70{ }^{\circ} \mathrm{C}^{[4]}$. The experimental results showed that tartary buckwheat polysaccharide had obvious inhibitory effect on superoxide anion ${ }^{[5]}$. Some studies have evaluated its DPPH free radicals, hydroxyl radicals and total reducing ability ${ }^{[6]}$, indicating that tartary buckwheat polysaccharide has good scavenging ability for hydroxyl radicals and DPPH free radicals ${ }^{[7]}$. The function and function of natural bioactive substances lay the foundation for the development and utilization of health food products ${ }^{[8]}$. 


\section{Pharmacological Action}

\subsection{Hypolipidemic}

Based on the establishment of hamster hyperlipidemia model ${ }^{[9]}$, the results showed that tartary buckwheat can not only change the liver pathological changes of model mice, but also inhibit the weight gain of model animals and reduce the level of serum biochemical indicators, while regulating its behavior. Learning characterization ${ }^{[10]}$. The mouse hyperlipidemia model was established and it was proved that the addition of tartary buckwheat starch in high-fat diet can effectively improve the impaired glucose tolerance caused by high-fat diet, and has better protection and repair effect on the antioxidant system in the body

\subsection{Lowering Blood Sugar}

A rat model of hyperglycemia was established by intraperitoneal injection of alloxan, and the bitter buckwheat flour or tartary buckwheat capsule was administered at the same time ${ }^{[11]}$. The results showed that the blood glucose concentration was significantly reduced ${ }^{[12]}$. Continuous administration of tartary buckwheat flavonoid extract to mice for 15 days can increase the glucose tolerance level of normal mice ${ }^{[13]}$, and significantly reduce the blood glucose level after 1 hour of sugar load $^{[14]}$.

\section{Clinical Application}

\subsection{Anti-cancer and Anti-cancer}

The magnesium rich in buckwheat can inhibit the development of cancer, help vasodilation, maintain normal function of the heart muscle, strengthen intestinal peristalsis, increase bile, and promote the body to eliminate waste. Studies have shown that buckwheat protein extract can inhibit the development of breast cancer by lowering serum estriol content ${ }^{[15]}$.

\subsection{Anti-aging}

Tartary buckwheat has high radiation protection properties and is an excellent therapeutic food for patients with radiation sickness. Tartary buckwheat fat has the effect of preventing freckles and senile plaques. After identification of the components, it was found that the leaves of tartary buckwheat contain highly active antioxidant enzymes such as SOD.

\section{Comprehensive Utilization}

At present, studies have shown that there are also active ingredients in tartary buck, tartary buckwheat and tartary buckwheat ${ }^{[16]}$.It is considered to use tartary buckwheat flavonoids as a raw material resource for the development of antitumor drugs or functional foods ${ }^{[17]}$. In addition, the results indicate that the antioxidant components in the tartary buckwheat shell have obvious DNA damage protection and can be used as a potential natural DNA protectant in food, medicine and other fields.In addition, bitter and deep-processed products not only have the effects of lowering blood sugar, protecting the liver, preventing coronary heart disease, etc., so that the active ingredients in bitter buckwheat help to absorb and enhance the efficacy.

\section{Discussion}

In recent years, with the deep analysis and research on medicinal plant resources, it has been found that in addition to possessing medicinal functions, bitter buckwheat can be used as a medicine and food homologue for food therapy and health, and can be widely used; After the preparation of bitter noodles, bitter biscuits, bitter powder, bitter glutinous rice, bitter buckwheat drink, increased bitterness of the edible form. When bitterness is used, the shells and leaves are often removed, and the seeds are applied. The waste generated during the processing can be applied to veterinary feed, 
promote digestion of the gastrointestinal tract of animals, and prevent animal diseases. Occurs; making the buckwheat shell into a pillow, increasing the comfort of the pillow, while the scent of bitterness helps sleep. There are many applicable parts in bitterness, and the application potential is developed to promote the sustainable development of traditional Chinese medicine while protecting medicinal resources, so as to bring broad social and economic benefits.

\section{Acknowledgements}

The article is supported by National International Cooperation Base (2016-151), Central Plains Scholar (162101510003) funds project.

\section{References}

[1] You Lingling, Liu Huanhua, Li Xiaoyan, et al. Purification and antioxidant activity of tartary buckwheat flavonoids[J]. Journal of the Chinese Cereals and Oils Association, 2014, 29(08): 23-27.

[2] Wang Sihui, Bai Yinhua, Huang Yuling, et al. Inhibition of $\alpha$-glucosidase by tartary buckwheat flavonoids[J].Food Science and Technology,2012,37(02):24-26+31.

[3] LI Fuhua, LIU Dong, MENG Jian. Antioxidant and antitumor activities of flavonoids from tartary buckwheat bran [J].Food Science, 2014, 35(07):58-63.

[4] Qiu Shuo. Extraction and Antioxidant Analysis of Tartary Buckwheat Polysaccharide [J].Diabetes and Health Research, 2015, 32(06):39-40.

[5] YANG Hongyan, YAN Nannan, JIANG Yanhong, et al. In vitro antioxidant activity and purification of polysaccharides from tartary buckwheat tea $[\mathrm{J}]$. Food and Fermentation Industries, 2014, 40(11): 94-99.

[6] LI Wei, CUI Xiaodong, LI Chen, et al. Antioxidation of Tartary Buckwheat Polysaccharide and Its Inhibition of Hep G2 Cell Proliferation[J].Food Research and Development,2016,37(01):9-12.

[7] Xu Qinglian, Guo Xing, Jiang Zijing, et al. Analysis and Identification of Aroma Components of Tartary Buckwheat Tea from Different Producing Areas by HS-SPME-GC-MS[J].Food and Fermentation Industries, 2017,43(08):233 -239.

[8] Yu Li, Wang Zhuozhen, Cheng Jianghua, et al.SDE/SPME-GC-MS analysis of volatile aroma components of tartary buckwheat[J].China Brewing,2015,34(02):148-152.

[9] Zhou Xiaoli, Lu Yuan, Zhou Ming, et al. Effects of tartary buckwheat protein on probiotic growth and its cholate tolerance [J]. Modern food science and technology, 2016, 32(09): 121-126.

[10] Zhang Zesheng, Yang Shuai, Lei Mengmeng, et al. Effects of whole leaves and alcohol extracts of Tartary buckwheat on blood lipids in hyperlipidemia hamsters[J].Food Research and Development,2014,35(18):83- 86.

[11] Zhou Xiaoli, Wang Yue, Zhao Wei, et al. Effects of Tartary Buckwheat on Blood Lipid Metabolism and Tissue Redox in Mice Induced by High-fat Diet[J]. Food Science, 2018, 39(03): 188-192.

[12] Zhou Xiaoli, Liu Taizhen, Yan Beibei, et al. Effects of tartary buckwheat on physiological and intestinal flora in mice induced by high fat diet[J].Food Science,2018,39(01):172-177.

[13] LIN Bing, HU Changling, HUANG Fang, et al. Research progress in chemical constituents and pharmacological activities of tartary buckwheat[J].Drugs \& Clinic,2011,26(01):29-32.

[14] ZHU Rui, GAO Nannan, CHEN Jianmin. Chemical Composition and Pharmacological Action of Tartary Buckwheat [J]. Chinese Wild Plant Resources, 2003(02):7-9.

[15] Hou Xuemei, Yuan Zhong. Nutritional health function and development and utilization of buckwheat [J]. Agricultural product processing (school), 2014(01):73-75. 
[16] LI Fuhua, LIU Dong, et al. Antioxidant and antitumor activities of flavonoids from tartary buckwheat bran[J].Food Science,2014,35(07):58-63.

[17] LI Fuhua,ZHANG Xiaoli,MING Jian.Study on the inhibition of proliferation of human colon cancer cell Caco-2 by tartary buckling polyphenols[J].Journal of the Chinese Cereals and Oils Association, 2015,30(11):31-36.

Miao Mingsan, male, professor, doctor, mainly engaged in Chinese medicine pharmacology teaching and research. Email: miaomingsan@163.com. 